\title{
Suppression of Large Edge-Localized Modes in High-Confinement DIII-D Plasmas with a Stochastic Magnetic Boundary
}

\author{
T. E. Evans, ${ }^{1}$ R. A. Moyer, ${ }^{2}$ P. R. Thomas, ${ }^{3}$ J. G. Watkins, ${ }^{4}$ T. H. Osborne, ${ }^{1}$ J. A. Boedo, ${ }^{2}$ E. J. Doyle, ${ }^{9}$ \\ M. E. Fenstermacher, ${ }^{5}$ K. H. Finken, ${ }^{6}$ R. J. Groebner, ${ }^{1}$ M. Groth, ${ }^{5}$ J. H. Harris, ${ }^{7}$ R. J. La Haye,${ }^{1}$ C. J. Lasnier, ${ }^{5}$ \\ S. Masuzaki, ${ }^{8}$ N. Ohyabu, ${ }^{8}$ D. G. Pretty, ${ }^{7}$ T. L. Rhodes, ${ }^{9}$ H. Reimerdes, ${ }^{10}$ D. L. Rudakov, ${ }^{2}$ M. J. Schaffer, ${ }^{1}$ \\ G. Wang, ${ }^{9}$ and L. Zeng ${ }^{9}$ \\ ${ }^{1}$ General Atomics, San Diego, California, USA \\ ${ }^{2}$ University of California San Diego, La Jolla, California, USA \\ ${ }^{3}$ CEA Cadarache Euratom Association, Cadarache, France \\ ${ }^{4}$ Sandia National Laboratories, Albuquerque, New Mexico, USA \\ ${ }^{5}$ Lawrence Livermore National Laboratory, Livermore, California, USA \\ ${ }^{6}$ FZ-Jülich Euratom Association, Jülich, Germany \\ ${ }^{7}$ Australian National University, Canberra, Australia \\ ${ }^{8}$ National Institute for Fusion Science, Gifu-ken, Japan \\ ${ }^{9}$ University of California, Los Angeles, California, USA \\ ${ }^{10}$ Columbia University, New York, New York, USA \\ (Received 10 December 2003; published 10 June 2004)
}

\begin{abstract}
A stochastic magnetic boundary, produced by an applied edge resonant magnetic perturbation, is used to suppress most large edge-localized modes (ELMs) in high confinement ( $H$-mode) plasmas. The resulting $H$ mode displays rapid, small oscillations with a bursty character modulated by a coherent $130 \mathrm{~Hz}$ envelope. The $H$ mode transport barrier and core confinement are unaffected by the stochastic boundary, despite a threefold drop in the toroidal rotation. These results demonstrate that stochastic boundaries are compatible with $H$ modes and may be attractive for ELM control in next-step fusion tokamaks.
\end{abstract}

DOI: $10.1103 /$ PhysRevLett.92.235003

PACS numbers: 28.52.-s, 52.55.Fa, 52.55.Rk

The physics of complex stochastic magnetic field configurations holds significant implications for research in astrophysical [1], solar [2], planetary [3], and laboratory [4] plasmas. In fusion plasmas, the tight coupling between fusion power production and plasma edge conditions (the height of the $H$-mode pedestal) leads to edge pressure gradients that generally produce large, repetitive heat and particle loading to the divertor targets known as edge-localized modes (ELMs) [5]. These ELMs are governed by the edge magnetohydrodynamic (MHD) stability, limit the core plasma performance, and reduce the divertor target plate lifetime due to increased erosion from the impulsive heat and particle fluxes [6]. However, the ELMs also transport heat and particles across the boundary, providing steady-state, high performance operations with density control and reduced core impurity content. Consequently, any technique to eliminate large ELM impulses while maintaining $H$ mode edge pedestal conditions must replace the ELM-induced transport with another, less transient, transport process. Such a technique would be highly desirable for a burning plasma experiment such as the proposed International Tokamak Experimental Reactor (ITER) device. Previous experiments have induced ELMs [7,8], but a better solution is to suppress or disperse the ELMs. A stochastic boundary might reduce large, fast ELM impulses by limiting the edge pedestal conditions while replacing the ELM transport with steady-state transport along the stochastic mag- netic field lines. Stochastic magnetic layers have been studied under a variety of tokamak plasma conditions [4], but these studies have not included poloidally diverted $H$-mode plasmas with large ELMs. In this Letter, we report the first such experiments showing that large ELMs can be suppressed with a stochastic boundary layer without degrading the quality of the $H$-mode confinement.

Steady-state $H$-mode plasmas without large amplitude ELMs except for a few isolated events have been produced in the DIII-D tokamak by applying small resonant magnetic field perturbations. In these plasmas, applied perturbations with amplitudes less than $0.25 \%$ of the equilibrium field convert large amplitude ELMs, seen in the particle recycling signals, into small amplitude intermittent bursts modulated by a coherent $130 \mathrm{~Hz}$ periodic oscillation. The $H$-mode radial transport barrier is not affected by the magnetic perturbations and electron pedestal profiles are not significantly changed. These results demonstrate that stochastic boundary layers are compatible with $H$-mode plasmas and that a stochastic layer can suppress ELMs without degrading the global particle and energy confinement.

The magnetic perturbations are produced by a set of control coils, referred to as the I coil, located inside the vacuum vessel [9]. The DIII-D error field correction coil (C coil) was not used in this experiment, since, in addition to correcting a known field error at the $q=2$ surface, it also 


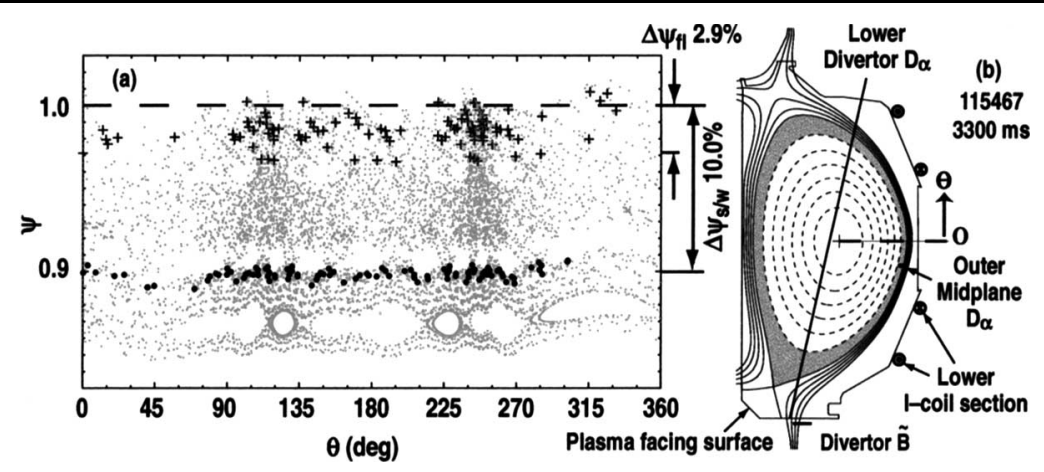

FIG. 1. (a) Poincaré plot of the edge magnetic field topology in the $(\psi, \theta)$ plane calculated by TRIP3D using the known intrinsic field errors and an $n=3,4.4 \mathrm{kA}$ I-coil perturbation with $0^{\circ}$ toroidal phase. $\psi$ is the normalized poloidal magnetic flux (a radial coordinate) and $\theta$ is the poloidal angle indicated in (b). The unperturbed separatrix is indicated with a dashed line at $\psi=1$. The black + symbols in the region $0.97<\psi<1$ show the innermost magnetic field line which is lost by crossing the separatrix into the region $\psi>1$. The black symbols near $\psi=0.9$ show the innermost magnetic field line which is stochastic without being lost from the plasma. (b) The unperturbed equilibrium reconstruction from the EFIT Grad-Shafranov solver showing $\psi$ contours (dashed ovals) and the TRIP3D computational domain of (a) as a shaded region at the edge of the plasma.

perturbs the boundary [10]. The I coil consists of 12 single-turn loops, six above and six below the midplane [Fig. 1(b)]. Each loop is constructed in a window pane geometry and mounted behind protective graphite tiles on the low field side of the vessel. The loops are up-down symmetric with respect to the midplane. For the experiments discussed here, the upper and lower loops had a toroidal mode number $n=3$ with opposite current polarities. This configuration results in an up-down asymmetric perturbation.

Field line integration modeling [10] of the I-coil perturbation [Fig. 1(a)] indicates that a narrow poloidal flux $(\psi)$ loss region, with a width $\Delta \psi_{\mathrm{fl}}=2.9 \%$, is formed just inside the unperturbed separatrix along with a weakly stochastic zone containing remnant islands and field lines that randomly traverse small regions of unperturbed poloidal flux. The width of the stochastic region $\Delta \psi_{\text {slw }}=$ $10.0 \%$, as seen from Fig. 1(a). Here, all the known sources of intrinsic field errors [11] are included.

The plasmas in this experiment were double null diverted discharges with a $2 \mathrm{~cm}$ downward shift [Fig. 1(b)]. The discharges had a major radius $\left(R_{0}\right)$ of $1.722 \mathrm{~m}$, an outer midplane minor radius $(a)$ of $0.585 \mathrm{~m}$, an elongation $(\kappa)$ of 1.8, and upper and lower triangularities of 0.35 and 0.73 , respectively. The toroidal magnetic field $\left(B_{T}\right)$ was $1.6 \mathrm{~T}$, with a plasma current $\left(I_{p}\right)$ of $1.1 \mathrm{MA}$, a neutral beam heating power $\left(P_{\text {inj }}\right)$ of $5.1 \mathrm{MW}$, and a safety factor at the $95 \%$ flux surface $\left(q_{95}\right)$ of 3.7. A typical ELM suppression discharge with $4.4 \mathrm{kA}$ in the I coil is compared to an identical discharge without the I-coil pulse in Fig. 2. The electron pedestal density normalized to the Greenwald density [12] was 0.55 with a line average density of $7.2 \times 10^{19} \mathrm{~m}^{-3}$ [Fig. 2(b)]. These discharges went into an $H$ mode at about $1600 \mathrm{~ms}$ and established regular type I ELMs within 300 to $400 \mathrm{~ms}$ after the $L-H$ transition [Figs. 2(c) and 2(d)]. Within one ELM cycle (about $15 \mathrm{~ms}$ ) after switching on the I coil at $t=3000 \mathrm{~ms}$ [Fig. 2(a)], the large type I ELM behavior is substantially reduced except for a few isolated events [Fig. 2(d)]. The ELM behavior in the discharge without an I-coil pulse is shown in Fig. 2(c). During the I-coil pulse, the discharge remains in $H$ mode and the core confinement, as indicated by the plasma stored energy, is the same as the reference discharge.

The suppression of type I ELM impulses is seen globally on all the diagnostics used to monitor ELM properties, including the $D_{\alpha}$ signals monitoring the outboard midplane [Fig. 3(a)] and the lower divertor [Fig. 3(b)], the particle flux to the divertor target plate, measured by Langmuir probes near the divertor strike point [Fig. 3(c)], and the surface temperature measured by an infrared TV camera in line-scan mode with a time resolution of $100 \mu \mathrm{s}$ [Fig. 3(d)]. In addition, there is a relatively slow $(\tau \approx 300 \mathrm{~ms}$ ) decay of the toroidal rotation [Fig. 3(e), black] compared to the reference discharge without the I-coil pulse [Fig. 3(e), gray]. In Fig. 3(f), a magnetic

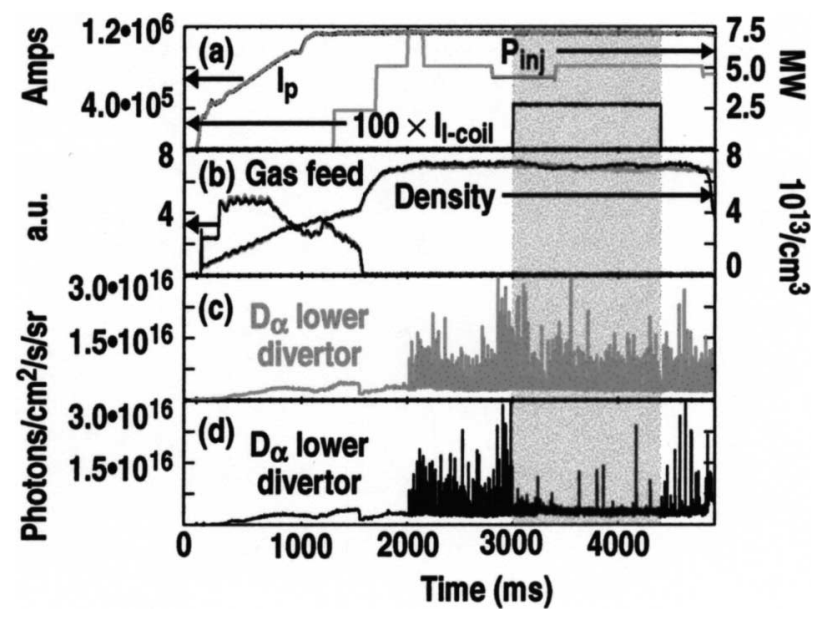

FIG. 2. Comparison of two identical discharges with and without I-coil current: (a) $I_{p}, P_{\text {inj }}$, and I-coil current; (b) gas feed and line integrated electron density; and recycling in the lower divertor (c) without and (d) with the I-coil pulse. 


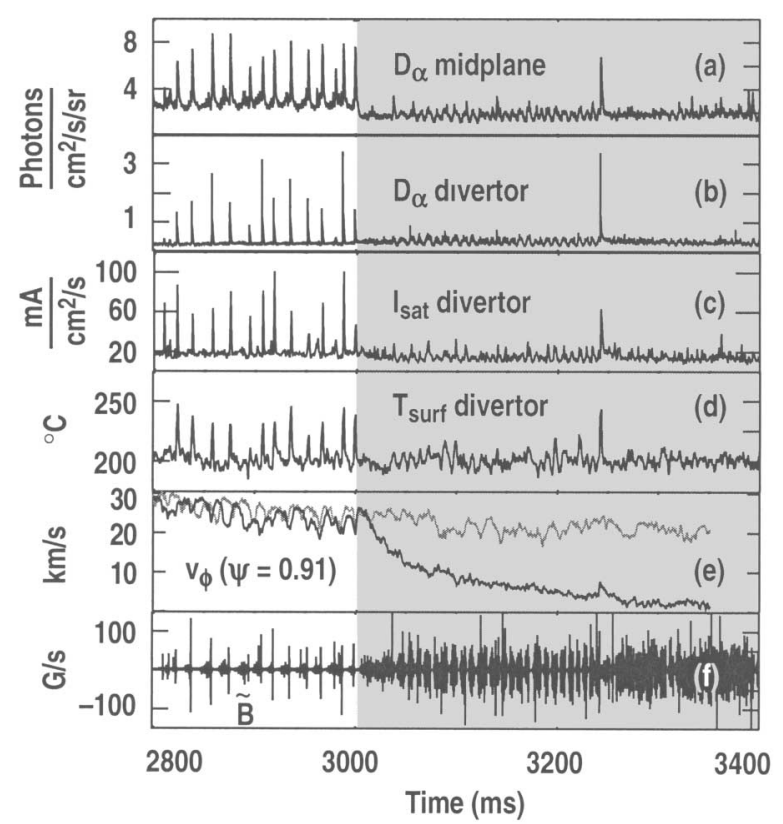

FIG. 3. ELM suppression during discharge 115467 (Fig. 2) using the I-coil parameters of Fig. 1 . The $D_{\alpha}$ recycling response from midplane (a) and lower divertor (b) chords are shown with the particle flux to a Langmuir probe (c) and the surface temperature from an IR camera view (d) near the outer strike point in the lower divertor. The edge toroidal rotation (e) is shown with (black) and without (gray) the I coil. Also shown is the lower divertor (f) magnetic fluctuation (Mirnov) signal. The shaded region indicates the time when the I coil is pulsed on with a current of $4.4 \mathrm{kA}$.

probe near the lower divertor strike point, which records edge MHD fluctuations without contributions from the core MHD modes, shows an increase in the bursty fluctuation behavior with a low frequency $(130 \mathrm{~Hz})$ modulation that switches the fluctuations on and off. A similar behavior is seen in the density fluctuations measured by the midplane reflectometers and in the particle flux measured by a reciprocating Langmuir probe array on the outer midplane several centimeters outside the unperturbed separatrix.

The resonant character of the ELM suppression was verified in discharges where $q_{95}$ was varied by ramping the $I_{p}$ during the I-coil pulse. Using discharges with the same parameters as in Fig. 2, I-coil pulses were triggered $300 \mathrm{~ms}$ before starting the $q_{95}$ ramp. The ELM suppression was maximized for $3.5 \leq q_{95} \leq 4.0$, indicating a resonant response to the applied perturbation.

The core performance is unaffected by the I coil. The plasma stored energy is unchanged, while the normalized plasma pressure $\beta_{N}=\beta a B / I_{p}=2.2$ and the $H$-mode quality factor $H_{L 89}=2.1$ remain constant. In addition, the gas fueling rate drops to zero when the plasma transitions into the $H$ mode [Fig. 2(b)] in both discharges. The total power radiated from the plasma core is also unaffected by the I-coil pulse. Since the plasma reaches a similar steady state with the same input power but with- out the impulsive particle and heat transport associated with the ELMs, the average radial transport during the I-coil pulse must be near the level obtained in the ELM phase. Measurements show that the magnetic field, density, and potential fluctuation levels increase during the ELM suppression indicating that the impulsive transport associated with the ELMs is replaced by an increase in turbulent transport in conjunction with parallel transport along the open stochastic field lines.

The $H$-mode pedestal height and radial transport barrier are unchanged during the I-coil pulse. A small $(\delta \psi=$ $1.5 \%$ ) outward shift in the time average pedestal electron pressure is seen during the I-coil pulse [Fig. 4(a)]. The ion channel, however, shows changes that might affect edge stability, including an increase in the pedestal carbon VI (CVI) ion pressure [Fig. 4(b)] as measured with charge exchange recombination spectroscopy. The pedestal ion temperature $T_{i}$ profile also broadens, suggesting that the main ion pressure profile broadens. The radial electric field $E_{r}$ is unchanged. The impact of these profile changes on the edge MHD stability is under investigation.

The $130 \mathrm{~Hz}$ oscillations seen on the particle flux [Fig. 3(c)] and recycling signals [Figs. 3(a) and 3(b)] during the I-coil pulse correlate with a periodic 1-2 ms expansion of the plasma during the low recycling phase followed by a $6 \mathrm{~ms}$ contraction of the plasma when the recycling increases. The recycling signals become significantly more bursty in character during the contraction phase of the cycle. Based on the changes in outer midplane separation with the wall, the amplitude of the oscillation is about $0.9 \%$ in $\psi$ and is seen globally on all the $D_{\alpha}$ recycling signals. There is a corresponding decrease in plasma stored energy of 5 to $10 \mathrm{~kJ}$ during each contraction, compared to a stored energy loss of 15 to $20 \mathrm{~kJ}$ during each of the type I ELM impulses. These oscillations, while lower in amplitude and about a factor of 3 longer in time, are similar to those seen during the type-I ELMs prior to the I-coil pulse. During the I-coil pulse, it appears that the plasma establishes a new dynamical state in which the integrated transport during one cycle of these oscillations is roughly equivalent to

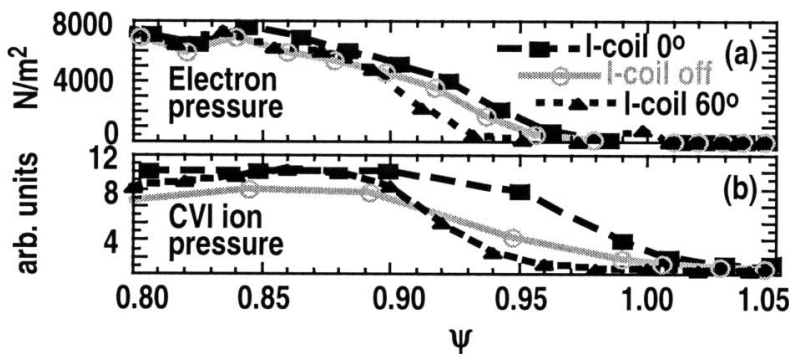

FIG. 4. (a) Electron pressure $P_{e}$ and (b) CVI impurity pressure $P_{\mathrm{CVI}}$ profiles with (black dashed lines) and without (gray solid lines) the I-coil perturbation. The reference discharge (circles) and two discharges with I-coil pulses having $0^{\circ}$ toroidal phase (solid squares) and $60^{\circ}$ toroidal phase (solid triangles) are shown. 


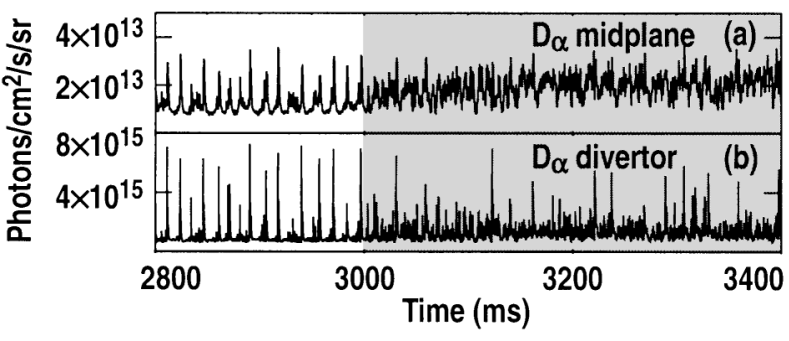

FIG. 5. (a) Midplane and (b) lower divertor recycling signals during a discharge with the toroidal phase of the I-coil perturbation rotated $60^{\circ}$ with respect to the phasing used in the ELM suppression discharge in Figs. 2 and 3. The shaded region indicates when the I coil is on.

that during a single type I ELM spike but extended over a longer time.

The toroidal phase of the I-coil perturbation was rotated $60^{\circ}$ in an identical discharge to determine if intrinsic field errors play a significant role in the plasma response. In contrast to the $0^{\circ}$ case, the $60^{\circ}$ phase resulted in a smaller effect on the ELMs [Fig. 5(b)], although the suppression is still quite significant. The $H$-mode pedestal response was also quite different: the edge electron pressure dropped just inside the separatrix [dashed black line in Fig. 4(a)] while the pressure at the top of the pedestal near $\psi=0.9$ remained constant. These changes suggest a small $\delta \psi=1.5 \%$ outward shift in the pedestal profile for the $0^{\circ}$ phasing and a larger $\delta \psi=5 \%$ inward shift for the $60^{\circ}$ phasing. The ion pressure [Fig. 4(b)] also changes in the $60^{\circ}$ case compared to the $0^{\circ}$ case. Because the ion and electron pressure profiles are measured at different toroidal and poloidal locations, these results suggest that the difference is a global effect and that a different stochastic boundary structure exists for these two cases. In addition, during the $60^{\circ}$ case the overall recycling level between ELMs measured at the outer midplane [Fig. 5(a)] increased until individual ELMs were no longer distinguishable at the outer midplane. The apparent elimination of ELMs on the midplane recycling measurements, in conjunction with a significant reduction in the frequency and amplitude of ELMs in the divertor [Fig. 5(b)], are suggestive of the enhanced $D_{\alpha}$ (EDA) mode in the C-Mod tokamak [13]. A possible interpretation is that the intrinsic field errors, due to coil misalignments, bus bars, etc., or even due to the magnetic field of the quasicoherent mode, might produce a stochastic layer in C-Mod sufficient to contribute to ELM suppression.

Although no change in the core confinement was observed in the $60^{\circ}$ case, the effect of the I-coil perturbation is consistent with a broader stochastic layer compared to the $0^{\circ}$ case. These observations are consistent with a broadening of the flux loss region that could be explained by the existence of an unknown "field error" that mixes differently with the two I-coil phases. In contrast, modeling with the field line integration code, using all the known field errors predicts that $\Delta \psi_{\text {slw }}$ and
$\Delta \psi_{\mathrm{fl}}$ are slightly smaller for the $60^{\circ}$ case. These results suggest that additional field errors are present and alter the effectiveness of the ELM suppression.

In conclusion, most large type I ELMs have been suppressed by applying an edge magnetic perturbation that produces a weakly stochastic boundary without affecting the core confinement or the $H$-mode transport barrier. The effectiveness of the ELM suppression depends on the toroidal phase of the applied perturbation and $q_{95}$. This is consistent with a resonant response to the applied perturbation. The results suggest that field errors play a role in the ELM behavior in a particular device and that "intrinsic" field errors in DIII-D are a significant factor in determining the ELM behavior. Because these field errors alter the magnetic topology in the pedestal region, understanding ELM stability and developing a predictive model for the $H$-mode pedestal will likely require an increased understanding of field errors and the plasma response to them. Finally, these results were obtained in double null discharges optimized for advanced tokamak operations, a regime that is "close" to conditions (e.g., high triangularity) for achieving small type II ELMs in other devices [14]. It is not clear if the suppression of large type I ELMs can also be achieved in low triangularity shapes. Future experiments in DIII-D will explore this possibility and may eventually result in an ELM suppression scenario for fusion reactors with more conventional tokamak shapes such as ITER.

This work was supported by the U.S. Department of Energy under Contracts No. DE-AC03-99ER54463, No. DE-AC04-94AL85000, and No. W-7405-ENG-48 and Grants No. DE-FG03-95ER54294, No. DE-FG0301ER54615, and No. DE-FG02-89ER54297.

[1] B. J. Albright, Phys. Plasmas 6, 4222 (1999).

[2] P. Mininni, D. Gómez, and B. Mindlin, Sol. Phys. 201, 203 (2001).

[3] F. Spahn et al., J. Geophys. Res. 108, 2-1 (2003).

[4] Ph. Ghendrih, A. Grosman, and H. Caps, Plasma Phys. Controlled Fusion 38, 1653 (1996).

[5] A. Loarte et al., Plasma Phys. Controlled Fusion 38, 1815 (2002).

[6] G. Federici et al., J. Nucl. Mater. 266-269, 109 (2003).

[7] M. Mori et al., Proceedings of the 14th IAEA Conference on Plasma Physics and Controlled Fusion, Würzburz (IAEA, Vienna, 1993), Vol. 2, p. 567.

[8] P.T. Lang et al., Nucl. Fusion 43, 1110 (2003).

[9] G. L. Jackson et al., Proceedings of the 30th European Physical Society Conference on Controlled Fusion and Plasma Physics, St. Petersburg, 2003 (European Physical Society, Geneva, 2003), CD-ROM, paper P-4.47.

[10] T. E. Evans, R. A. Moyer, and P. Monat, Phys. Plasmas 9, 4957 (2002).

[11] J. L. Luxon et al., Nucl. Fusion 43, 1813 (2003).

[12] M. Greenwald et al., Nucl. Fusion 28, 2199 (1988).

[13] S. J. Wukitch et al., Phys. Plasmas 9, 2149 (2002).

[14] J. Stober et al., Nucl. Fusion 41, 1123 (2001). 